Internat. J. Math. \& Math. Sci.

Vol. 24, No. 2 (2000) 129-137

S0161171200002428

(C) Hindawi Publishing Corp.

\title{
THEORETICAL SCHEME ON NUMERICAL CONFORMAL MAPPING OF UNBOUNDED MULTIPLY CONNECTED DOMAIN BY FUNDAMENTAL SOLUTIONS METHOD
}

\author{
TETSUO INOUE, HIDEO KUHARA, KANAME AMANO, and DAI OKANO
}

(Received 8 December 1997)

\begin{abstract}
A potentially theoretical scheme in the fundamental solutions method, different from the conventional one, is proposed for numerical conformal mappings of unbounded multiply connected domains. The scheme is introduced from an algorithm on numerical Dirichlet problem, based on the asymptotic theorem on extremal weighted polynomials. The scheme introduced in this paper has the characteristic called "invariant and dual."
\end{abstract}

Keywords and phrases. Extremal weighted polynomial, fundamental solutions method, unbounded multiply connected domain, numerical conformal mapping, invariant and dual.

2000 Mathematics Subject Classification. Primary 30E10, 41A10, 65 E05.

1. Introduction. The fundamental solutions method (or charge simulation method) has been applied to the problem in electrical engineering, numerical conformal mappings [2, 3, 4, 6] and Dirichlet problems [8, 9, 15, 16].

The principle of the method is the approximation of the solution by a linear combination of logarithmic potentials. Though the method requires only solving a system of simultaneous linear equations, it is possible to get a rather precise solution for boundary problems with respect to domains bounded by smooth curves.

In this paper, we study the fundamental solutions method for numerical conformal mappings of unbounded multiply connected domains. The new scheme is theoretically proposed applying the algorithm on numerical Dirichlet problem based on the asymptotic theorems $[7,12,13,14]$ on extremal weighted polynomials.

Amano [2, 3] has recently proposed two kinds of schemes of approximations for the conformal mappings onto the domains with circular or radial cuts, respectively. The scheme introduced in this paper is applicable for both of above domains and has the characteristic called "invariant and dual."

Kuhara $[10,11]$ has also established a construction method of the functions mapping multiply connected domains onto the rings with circular or radial slits, based upon the works of Bergman [5] and using the fundamental solutions method. The method is described from the two-dimensional electrostatic point of view.

2. Scheme for numerical Dirichlet problem. An algorithm has been recently proposed for numerical Dirichlet problem of unbounded Jordan domains [8]. It is easily 


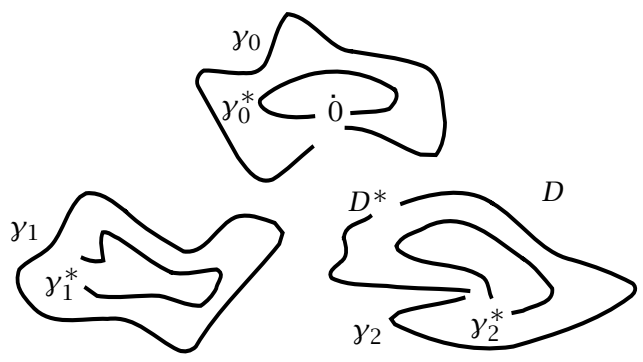

Figure 2.1. The domain $D$ and $D^{*}$.

transformed to multiply connected domains. For the convenience of readers, the outline is shown with a minor modification as follows.

At first we introduce the notions of weighted polynomials (shortly $w$-polynomials) and weighted capacity ( $w$-capacity) depending on the author [7] and Mhaskar-Saff $[13,14]$, respectively. The definitions of normalized counting measures and the weak convergence are also shown.

Let $D$ denote an unbounded domain whose boundary $\gamma$ consists of Jordan curves $\gamma_{i}(i=0(1) n)$. Without loss of generality, we assume that $D$ contains $\infty$ in its interior and $\gamma_{0}$ encloses the origin.

Let $w=w(z)$ be an arbitrary, continuous, positive function defined on $\gamma$. For each integer $n \geq 1$, we let $P_{n, w}$ denote the class of all polynomials of the form

$$
p_{n, w}(z)=\prod_{i=1}^{n}\left[\left(z-z_{n, i}\right) w(z) w\left(z_{n, i}\right)\right],
$$

which we call $w$-polynomials of degree $n$.

Let $M(\gamma)$ denote the class of all positive unit Borel measures whose support is $\gamma$. We define the $w$-energy of $\sigma \in M(\gamma)$ and the $w$-capacity by

$$
I_{w}(\sigma)=\iint \log [|z-t| w(z) w(t)] d \sigma(z) d \sigma(t)
$$

and

$$
\operatorname{cap}(w, \gamma)=\exp \left(V_{w}\right)
$$

respectively, where

$$
V_{w}=V(w, \gamma)=\sup _{\sigma \in M(\gamma)} I_{w}(\sigma) .
$$

We note that the notions of $w$-energy and $w$-capacity were introduced in [13, 14].

Let $\mu_{w} \in M(\gamma)$ be an extremal measure such that

$$
I_{w}\left(\mu_{w}\right)=V_{w} .
$$

The existence and the uniqueness of $\mu_{w}$ were shown in [14, Theorem 3.1(b)]. We assume that $S_{w}=\gamma$, where $S_{w}$ is support of $\mu_{w}$. 
Lastly, we show the notions of the normalized counting measure on the zeros and the weak convergence as follows: for $w$-polynomials $p_{n, w}(z)$ of degree $n$, the discrete unit measure defined on compact sets in the complex plane $C$ with mass $1 / n$ at each zero of $p_{n, w}(z)$ is denoted by $\mu_{n, w}=\mu\left(p_{n, w}\right)$. It is called the normalized counting measure on the zeros of $p_{n, w}(z)$. If $p_{n, w}(z)$ has multiple zeros, the obvious modification is considered.

The weak convergence of $v_{n}$ to $v$ as $n \rightarrow \infty$ is defined by

$$
\lim _{n \rightarrow \infty} \int f d v_{n}=\int f d v
$$

for every continuous function in the complex plane $C$ with compact support.

We present the fundamental lemma on extremal $w$-polynomials that shown in [8].

LEMMA 2.1. The necessary and sufficient condition that

$$
\lim _{n \rightarrow \infty}\left|\prod_{i=1}^{n}\left(z-z_{n, i}\right) w\left(z_{n, i}\right)\right|^{1 / n}=\exp \left\{\int \log [|z-t| w(t)] d \mu_{w}(t)\right\}
$$

holds uniformly on every compact subset of $D$ is:

(A) $\mu_{n, w}$ converges weakly to $\mu_{w}$ as $n \rightarrow \infty$, where $\mu_{n, w}=\mu\left(p_{n, w}\right)$ is the normalized counting measure of $p_{n, w}(z)=\prod_{i=1}^{n}\left[\left(z-z_{n, i}\right) w(z) w\left(z_{n, i}\right)\right]$.

Furthermore, if the condition $(A)$ is satisfied, the equality

$$
\frac{\exp \left\{\int \log [|z-t| w(t)] d \mu_{w}(t)\right\}}{\operatorname{cap}(w, \gamma)}=\frac{1}{w(z)}
$$

holds quasi-everywhere (q.e.) on $\gamma$ (we say that a property holds q.e. on $\gamma$ if the subset $\gamma^{\prime}$ of $\gamma$ where it does not hold has capacity zero).

Let $D$ and $D^{*}\left(D \subset D^{*}\right)$ be unbounded multiply connected domains with the boundaries $\gamma$ and $\gamma^{*}=\sum_{i=0}^{m} \gamma_{i}^{*}$, respectively. We assume that both $D$ and $D^{*}$ contain $\infty$ and zero in their interiors and exteriors, respectively (see Figure 2.1).

Let the function $H(z)$ be harmonic in $D^{*}$, where $H(z)=h(z)$ and $h^{*}(z)$ on $\gamma$ and $\gamma^{*}$, respectively. Then, we apply Lemma 2.1 for the domain $D^{*}$ and let

$$
w^{*}(z)=\exp \left\{-\left(h^{*}(z)+\log |z|\right)\right\}=\frac{\exp \left\{-h^{*}(z)\right\}}{|z|} .
$$

When the points $\left\{z_{n, i}\right\}_{i=1}^{n}$ on $\gamma^{*}$ satisfying the condition (A) are determined, the equalities

$$
H_{1}(z)=\log \lim _{n \rightarrow \infty} \frac{\prod_{i=1}^{n}\left|\left(z-z_{n, i}\right) w^{*}\left(z_{n, i}\right)\right|^{1 / n}}{\operatorname{cap}\left(w^{*}, \gamma^{*}\right)}=\int \log \frac{|z-t| w^{*}(t)}{\operatorname{cap}\left(w^{*}, \gamma^{*}\right)} d \mu_{w^{*}}(t)
$$

hold uniformly on every compact subset of $D^{*}$, which follows from Lemma 2.1.

Since $\gamma$ is a compact set in $D^{*}$, the convergence is uniform on $\gamma$. Furthermore, from (2.8),

$$
\frac{\exp \left\{\int \log \left[|z-t| w^{*}(t)\right] d \mu_{w^{*}}(t)\right\}}{\operatorname{cap}\left(w^{*}, \gamma^{*}\right)}=\frac{1}{w^{*}(z)}
$$


holds q.e. on $\gamma^{*}$. Combining (2.9), (2.10), and (2.11), the function

$$
H^{*}(z)=H_{1}(z)-\log |z|=\log \lim _{n \rightarrow \infty} \frac{\prod_{i=1}^{n}\left|\left(1-z_{n, i} / z\right) w^{*}\left(z_{n, i}\right)\right|^{1 / n}}{\operatorname{cap}\left(w^{*}, \gamma^{*}\right)}
$$

satisfies $H^{*}(z)=h^{*}(z)$ q.e. on $\gamma^{*}$. Since

$$
\lim _{z \rightarrow \infty} H^{*}(z)=\int \log \frac{w^{*}(t)}{\operatorname{cap}\left(w^{*}, \gamma^{*}\right)} d \mu_{w^{*}}(t)
$$

is finite, $H^{*}(z)$ is harmonic in $D^{*} \cup \infty[17]$.

Applying generalized Maximun Principle (two harmonic functions with q.e. same boundary values are equal to each other in the domain [17]) for the function $H(z)-$ $H^{*}(z)$, we obtain the equality $H(z)=H^{*}(z)$ in $D^{*} \cup \infty$.

Let $h(z)$ be a given function which is continuous on $\gamma$. The above argument suggests us the following algorithm for the fundamental solutions method of Dirichlet problem (i.e., to find the function $H(z)$ harmonic in $D \cup\{\infty\}$ such that $H(z)=h(z)$ on $\gamma$ ).

Algorithm 2.2. The approximation $H_{n}(z)$ of $H(z)$ is obtained as follows:

(i) Let $\left\{z_{n, i}\right\}_{i=1}^{n}$ (called charge points) and $\left\{\zeta_{n, i}\right\}_{i=1}^{n}$ (called collocation points) be appropriately chosen on $\gamma^{*}$ and $\gamma$, respectively.

(ii) When $\alpha_{i}(i=0,1,2, \ldots, n)$ are the solution of a system of simultaneous linear equations

$$
\alpha_{0}+\sum_{i=1}^{n} \alpha_{i} \log \left|1-\frac{z_{n, i}}{\zeta_{n, k}}\right|=h\left(\zeta_{n, k}\right) \quad(k=1,2, \ldots, n), \sum_{i=1}^{n} \alpha_{i}=1,
$$

the charges at $\left\{z_{n, i}\right\}_{i=1}^{n}$ are given by $\left\{\alpha_{i}\right\}_{i=1}^{n}$.

(iii) The approximation $H_{n}(z)$ is represented by

$$
H_{n}(z)=\alpha_{0}+\sum_{i=1}^{n} \alpha_{i} \log \left|1-\frac{z_{n, i}}{z}\right|
$$

If the charge points and the collocation points are "theoretically" chosen, we suppose that the approximations

$$
\alpha_{0} \simeq H(\infty), \quad \alpha_{i} \simeq \frac{1}{n} \quad(i=1,2, \ldots, n)
$$

hold.

Now, we consider the case when $D$ is a bounded multiply connected domain containing 0 and $\infty$ in its interior and exterior, respectively. Using the transformation $z \rightarrow 1 / z$, we propose the new scheme (to be called "dual") corresponding to (2.15) as follows:

$$
H_{n}(z)=\alpha_{0}+\sum_{i=1}^{n} \alpha_{i} \log \left|1-\frac{z}{z_{n, i}}\right|, \quad \sum_{i=1}^{n} \alpha_{i}=1
$$




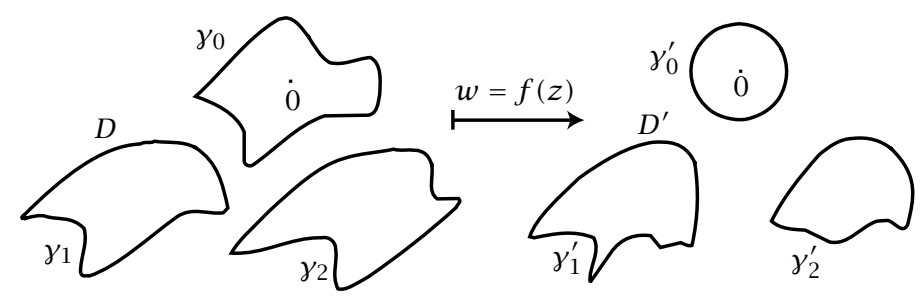

FIGURE 3.1. $f(z)$ mapping conformally $D$ onto $D^{\prime}$.

3. Scheme for numerical conformal mapping. Let $D$ and $D^{\prime}$ denote unbounded multiply connected domains whose boundaries $\gamma$ and $\gamma^{\prime}$ consist of Jordan curves $\gamma_{i}$ and $\gamma_{i}^{\prime}(i=0(1) m)$, respectively.

We assume that $\gamma_{0}, \gamma_{0}^{\prime}$ enclose the origin. Let $\gamma_{0}^{\prime}$ be a circle $\left\{w ;|w|=r_{0}\right\}$. Let $f(z)$ map conformally $D$ onto $D^{\prime}$ with the continuation to a bijection mapping from

$$
D \cup \gamma \rightarrow D^{\prime} \cup \gamma^{\prime}
$$

corresponding $\gamma_{i}$ to $\gamma_{i}^{\prime} \cdot f(z)$ is uniquely determined under the condition $f(\infty)=\infty$, $f^{\prime}(\infty)=1[1]$.

We propose the following scheme of approximations of $f(z)$ :

$$
f_{n}(z)=z \prod_{i=1}^{n}\left(1-\frac{z_{n, i}}{z}\right)^{\alpha_{i}}, \quad \sum_{i=1}^{n} \alpha_{i}=1,
$$

where the charge points $\left\{z_{n, i}\right\}_{i=1}^{n}$ are appropriately chosen interior to $\gamma$.

Algorithm 2.2 suggests us the scheme (3.2) for the approximation $f_{n}(z)$ of $f(z)$ in the fundamental solutions method. More precisely, we consider the equality

$$
\log \left|f_{n}(z)\right|=\log |z|+\sum_{i=1}^{n} \alpha_{i} \log \left|1-\frac{z_{n, i}}{z}\right|,
$$

which follows from (3.2).

Comparing (3.2) with (2.15), note that $\log \left|f_{n}(z)\right|$ has the term $\log |z|$ but the constant one $\alpha_{0}$. This is reasonable from the normalized condition $f(\infty)=\infty, f^{\prime}(\infty)=1$.

When $D^{\prime}$ is $\left\{w ;|w|>r_{0}\right\}$ with circular cuts $\sum_{i=1}^{m} \gamma_{i}^{\prime}$, we propose the algorithm computing approximations of $f(z)$ as follows.

Algorithm 3.1. The approximation $f_{n}(z)$ of $f(z)$ may be obtained as follows:

(i) $\left\{z_{n_{j}, i}^{(j)}\right\}_{i=1}^{n_{j}}$ and $\left\{\zeta_{n_{j}, i}^{(j)}\right\}_{i=1}^{n_{j}}$ with $\sum_{j=0}^{m} n_{j}=n$ are appropriately chosen interior to $\gamma_{j}$ and on $\gamma_{j}(j=0(1) m)$, respectively.

(ii) When $\alpha_{i}^{(j)}\left(i=0(1) n_{j}, j=0(1) m\right.$ ) are the solutions of a system of $n_{0}+\cdots+$ $n_{m}+m+1$ simultaneous linear equations assuming $n_{0}=\cdots=n_{m}=n$, and using 


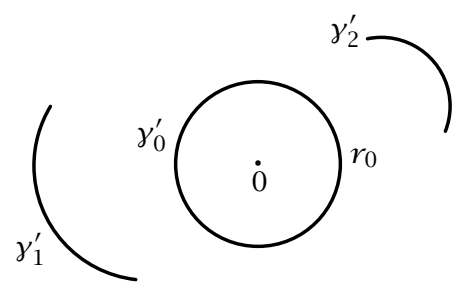

FIGURE 3.2. The domain $D^{\prime}$ with circular cuts.

Dirichlet and charge conditions [11]

$$
\begin{aligned}
\alpha_{0}^{(l)}+\log \left|\zeta_{n_{j}, k}^{(l)}\right|+ & \sum_{j=0}^{m} \sum_{i=1}^{n_{j}} \alpha_{i}^{(j)} \log \left|1-\frac{z_{n_{j}, i}^{(j)}}{\zeta_{n_{l}, k}^{(l)}}\right|=0 \quad\left(k=1(1) n_{l}, l=0(1) m\right), \\
& \sum_{i=1}^{n_{0}} \alpha_{i}^{(0)}=1, \quad \sum_{i=1}^{n_{j}} \alpha_{i}^{(j)}=0 \quad(j=1(1) m),
\end{aligned}
$$

the charges at $\left\{z_{n_{j}, i}^{(j)}\right\}_{i=1}^{n_{j}}$ are given by $\left\{\alpha_{i}^{(j)}\right\}_{i=1}^{n_{j}}(j=1(1) m)$, respectively.

(iii) The approximation $f_{n}(z)$ is represented by

$$
f_{n}(z)=z \prod_{j=0}^{m} \prod_{i=1}^{n_{j}}\left(1-\frac{z_{n_{j}, i}^{(j)}}{z}\right)^{\alpha_{i}^{(j)}}, \quad \sum_{i=1}^{n_{0}} \alpha_{i}^{(0)}=1, \quad \sum_{i=1}^{n_{j}} \alpha_{i}^{(j)}=0 \quad(j=1(1) m),
$$

equation (3.6) may be transformed to

$$
f_{n}(z)=\prod_{j=0}^{m} \prod_{i=1}^{n_{j}}\left(z-z_{n_{j}, i}^{(j)}\right)^{\alpha_{i}^{(j)}}, \quad \sum_{i=1}^{n_{0}} \alpha_{i}^{(0)}=1, \quad \sum_{i=1}^{n_{j}} \alpha_{i}^{(j)}=0 \quad(j=1(1) m),
$$

which implies that

$$
\int_{\gamma_{0}} d \arg f_{n}(z)=2 \pi, \quad \int_{\gamma_{j}} d \arg f_{n}(z)=0 \quad(j=1(1) m) .
$$

Note that

$$
\int_{\gamma_{0}} d \arg f(z)=2 \pi, \quad \int_{\gamma_{j}} d \arg f(z)=0 \quad(j=1(1) m)
$$

for the smooth boundaries $\gamma_{j}(j=0(1) m)$.

Note that the approximations

$$
\alpha_{0}^{(j)} \simeq-\log r_{j} \quad(j=0(1) m)
$$

hold, where $r_{j}$ is the radius of $\gamma_{j}^{\prime}$.

The solutions of a system of simultaneous linear equations in Algorithm 3.1 are invariant in the sense that the transformation $z \rightarrow a z(a>0)$ implies

$$
\begin{aligned}
& \alpha_{0}^{(j)} \longrightarrow \alpha_{0}^{(j)}+\log a \quad(j=0(1) m), \\
& \alpha_{i}^{(j)} \longrightarrow \alpha_{i}^{(j)} \quad(i=1(1) n, j=0(1) m) .
\end{aligned}
$$

Then, $f_{n}(z)$ is transformed to itself. 


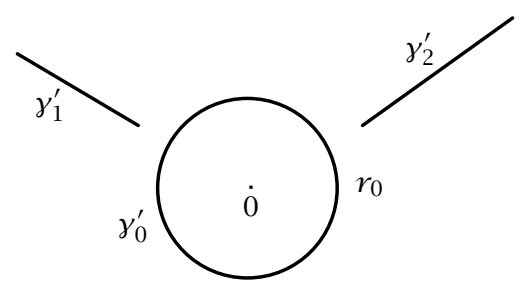

FIGURE 3.3. The domain $D^{\prime}$ with radial cuts.

The invariant scheme of approximations has been first shown for the numerical Dirichlet problem by Murota $[15,16]$. It is physically natural and mathematically reasonable.

When $D^{\prime}$ is $\left\{w ;|w|>r_{0}\right\}$ with radial cuts $\sum_{i=1}^{m} \gamma_{i}^{\prime}$, we propose the algorithm computing approximations of $f(z)$ as follows.

Algorithm 3.2. The approximation $f_{n}(z)$ of $f(z)$ may be obtained as follows:

(i) $\left\{z_{n_{j}, i}^{(j)}\right\}_{i=1}^{n_{j}}$ and $\left\{\zeta_{n_{j}, i}^{(j)}\right\}_{i=1}^{n_{j}}$ with $\sum_{j=0}^{m} n_{j}=n$ are appropriately chosen interior to $\gamma_{j}$ and on $\gamma_{j}(j=0(1) m)$, respectively.

(ii) When $\alpha_{i}^{(j)}\left(i=0(1) n_{j}, j=0(1) m\right)$ are the solutions of a system of $(m+1)(n+1)$ simultaneous linear equations using Dirichlet-Neumann and charge conditions [11, 10]:

$$
\begin{gathered}
\alpha_{0}^{(0)}+\log \left|\zeta_{n_{0}, k}^{(0)}\right|+\sum_{j=0}^{m} \sum_{i=1}^{n_{j}} \alpha_{i}^{(j)} \log \left|1-\frac{z_{n_{j}, i}^{(j)}}{\zeta_{n_{0}, k}^{(0)}}\right|=0 \quad\left(k=1(1) n_{0}\right), \\
\alpha_{0}^{(l)}+\arg \left|\zeta_{n_{l}, k}^{(l)}\right|+\sum_{j=0}^{m} \sum_{i=1}^{n_{j}} \alpha_{i}^{(j)} \arg \left(1-\frac{z_{n_{j}, i}^{(j)}}{\zeta_{n_{l}, k}^{(l)}}\right)=0 \quad\left(k=1(1) n_{l}, l=1(1) m\right), \\
\sum_{i=1}^{n_{0}} \alpha_{i}^{(0)}=1, \quad \sum_{i=1}^{n_{j}} \alpha_{i}^{(j)}=0 \quad(j=1(1) m),
\end{gathered}
$$

the charges at $\left\{z_{n_{j}, i}^{(j)}\right\}_{i=1}^{n_{j}}$ are given by $\left\{\alpha_{i}^{(j)}\right\}_{i=1}^{n_{j}}(j=1(1) m)$, respectively.

(iii) The approximation $f_{n}(z)$ is represented by

$$
f_{n}(z)=z \prod_{j=0}^{m} \prod_{i=1}^{n_{j}}\left(1-\frac{z_{n_{j}, i}^{(j)}}{z}\right)^{\alpha_{i}^{(j)}}, \quad \sum_{i=1}^{n_{0}} \alpha_{i}^{(0)}=1, \quad \sum_{i=1}^{n_{j}} \alpha_{i}^{(j)}=0 \quad(j=1(1) m) .
$$

The solutions of a system of simultaneous linear equations in Algorithm 3.2 are also invariant. Note that the approximations

$$
\alpha_{0}^{(0)} \simeq-\log r_{0}, \quad \alpha_{0}^{(j)} \simeq-\theta_{j} \quad(j=1(1) m)
$$

hold, where $\theta_{j}$ is the argument of $\gamma_{j}^{\prime}$.

The solutions of a system of simultaneous linear equations in Algorithm 3.2 are also invariant in the sense that the transformation $z \rightarrow a z(a>0)$ implies 


$$
\begin{aligned}
& \alpha_{0}^{(0)} \longrightarrow \alpha_{0}^{(0)}+\log a, \\
& \alpha_{0}^{(j)} \longrightarrow \alpha_{0}^{(j)} \quad(j=1(1) m), \\
& \alpha_{i}^{(j)} \longrightarrow \alpha_{i}^{(j)} \quad(i=1(1) n, j=0(1) m) .
\end{aligned}
$$

Then, $f_{n}(z)$ is transformed to itself.

4. Concluding remark. When $D$ and $D^{\prime}$ are a bounded multiply connected domain and $\left\{w ;|w|<r_{0}\right\}$ with circular or radial cuts, respectively, we propose the following scheme of approximations for the function $f(z)$ mapping $D$ onto $D^{\prime}$ with $f(0)=$ $0, f^{\prime}(0)=1$, considering the transformation $f(z) \rightarrow 1 / f(1 / z)$ :

$$
f_{n}(z)=z \prod_{j=0}^{m} \prod_{i=1}^{n_{j}}\left(1-\frac{z}{z_{n_{j}, i}^{(j)}}\right)^{\alpha_{i}^{(j)}}, \quad \sum_{i=1}^{n_{0}} \alpha_{i}^{(0)}=-1, \quad \sum_{i=1}^{n_{j}} \alpha_{i}^{(j)}=0 \quad(j=1(1) m) .
$$

The object of this paper is the study of the theoretical scheme based on the asymptotic theorem on extremal weighted polynomials, different from the conventional one, for the numerical conformal mapping onto $\left\{w ;|w|>r_{0}\right\}$ with circular or radial cuts. The scheme introduced in this paper is applicable for both of the above domains and has the characteristic called "invariant and dual." The numerical experiments in detail by the scheme will appear in a future paper.

\section{REFERENCES}

[1] L. V. Ahlfors, Complex Analysis: An Introduction of the Theory of Analytic Functions of One Complex Variable, McGraw-Hill Book Co., New York, 1966. MR 32\#5844. Zbl 154.31904.

[2] K. Amano, Numerical conformal mapping onto radial slit domains by the charge simulation method, Sūrikaisekikenkyūsho Kōkyūroku (1995), no. 915, 107-118. CMP 1385269.

[3] _ Numerical conformal mapping onto the circular slit domains, Trans. Inform. Process. Soc. Japan 36 (1995), no. 2, 219-225. CMP 1322479.

[4] A A charge simulation method for numerical conformal mapping onto circular and radial slit domains, SIAM J. Sci. Comput. 19 (1998), no. 4, 1169-1187 (electronic). MR 99b:30007. Zbl 907.30009.

[5] S. Bergman, The Kernel Function and Conformal Mapping, Amer. Math. Soc., New York, NY, 1950, Mathematical Survey, No. V. MR 12,402a. Zbl 040.19001.

[6] T. Inoue, Asymptotic behavior of extremal weighted polynomials, Mathematica (Cluj) 35(58) (1993), no. 1, 29-34. MR 95k:30010. Zbl 803.30005.

[7] _ Applications of asymptotic theorem on weighted extremal polynomials, Trans. Japan Soc. Ind. App. Math. 4 (1994), 151-155.

[8] _ Mathematical approach and numerical analysis to the fundamental solutions method of Dirichlet problem, Int. J. Math. Math. Sci. 22 (1999), no. 2, 349-365. MR 2000d:65046. Zbl 990.53224.

[9] M. Katsurada and H. Okamoto, The collocation points of the fundamental solution method for the potential problem, Comput. Math. Appl. 31 (1996), no. 1, 123-137. CMP 1362 387. Zbl 852.65101.

[10] H. Kuhara, S. Oshima, and K. Yasuda, A charge simulation for canonical conformal mapping of multiply connected regions onto rings with radial slits, Paper of Technical Meeting on Electromagnetic Theory, IEE and IEIC of Japan, 1997, EMT-97-83. 
[11] H. Kuhara, T. Yonezawa, and S. Oshima, Construction of transformations mapping multiply connected regions conformally onto the canonical ones using the charge simulation method (Japanese), Paper of Technical Meeting on Electromagnetic Theory, IEE and IEIC of Japan, 1996, EMT-96-110.

[12] H. N. Mhaskar, Finite-infinite-range inequalities in the complex plane, Internat. J. Math. Math. Sci. 14 (1991), no. 4, 625-638. MR 93b:26023. Zbl 774.30004.

[13] H. N. Mhaskar and E. B. Saff, Where does the sup norm of a weighted polynomial live? (A generalization of incomplete polynomials), Constr. Approx. 1 (1985), no. 1, 71-91. MR 86a:41004. Zbl 582.41009.

[14] Weighted analogues of capacity, transfinite diameter, and Chebyshev constant, Constr. Approx. 8 (1992), no. 1, 105-124. MR 93a:31004. Zbl 747.31001.

[15] K. Murota, On "invariance" of schemes in the fundamental solution method, Trans. Inform. Process. Soc. Japan. 3 (1993), 535-535.

[16] Comparison of conventional and "invariant" schemes of fundamental solutions method for annular domains, Japan J. Indust. Appl. Math. 12 (1995), no. 1, 61-85. MR 95k:65098. Zbl 831.65118.

[17] M. Tsuji, Potential Theory in Modern Function Theory, Chelsea Publishing Co., New York, 1975. MR 54\#2990. Zbl 322.30001.

TETSUO INOUE: DePARTMENT Of INFORMATION SYSTEMS ENGINEERING, KOBE MERCANTILE MARINE COLLEGE, KoBE, JAPAN

E-mail address: inoue-te@ti edu.kshosen.ac.jp

Hideo Kuhara: Yatsusiro National College of Technology, Kumamoto, JaPan

Kaname Amano and Dai Okano: Department of Computer Science, Faculty of EngiNEERING, EHIME UNIVERSITY, EHIME, JAPAN 


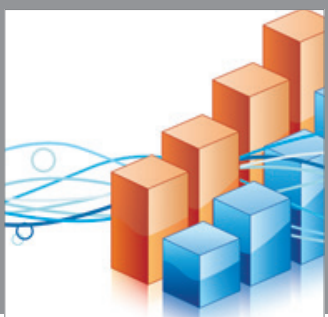

Advances in

Operations Research

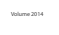

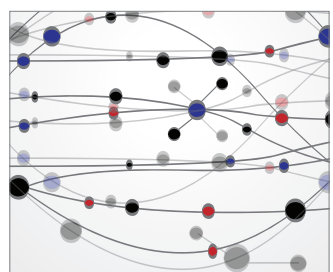

\section{The Scientific} World Journal
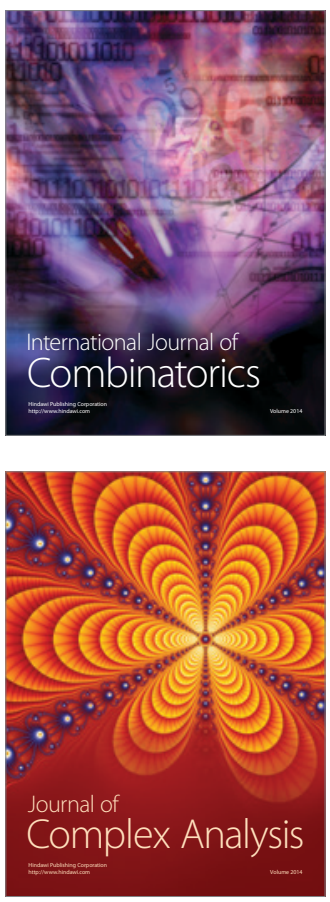

International Journal of

Mathematics and

Mathematical

Sciences
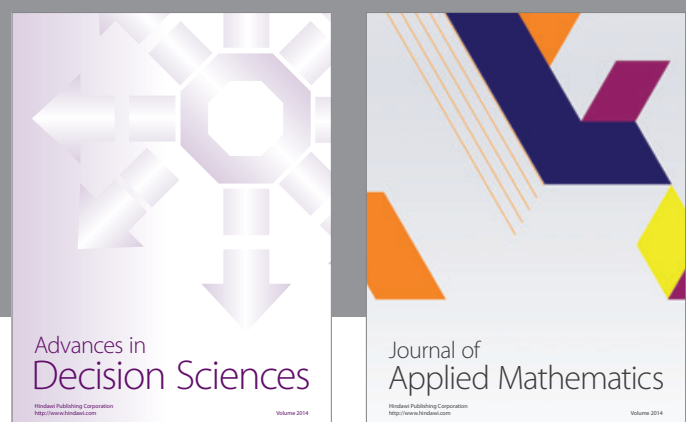

Journal of

Applied Mathematics
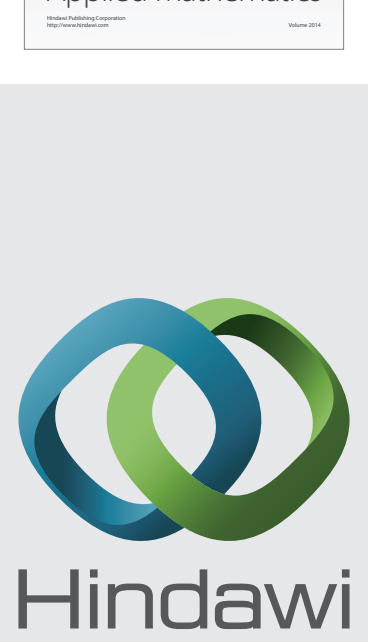

Submit your manuscripts at http://www.hindawi.com
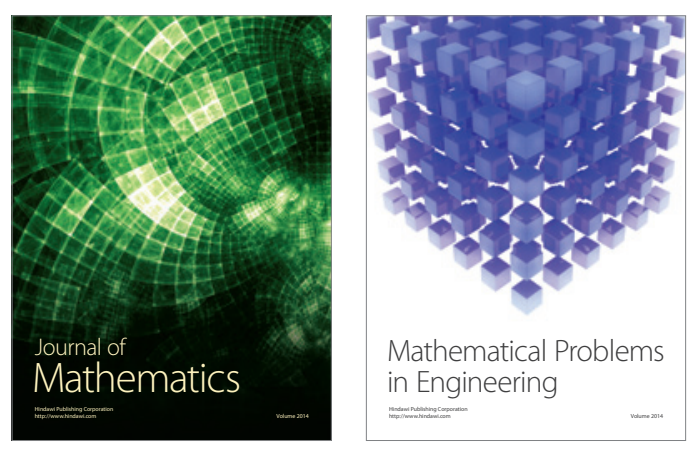

Mathematical Problems in Engineering
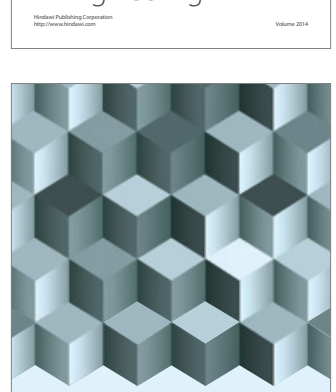

Journal of

Function Spaces
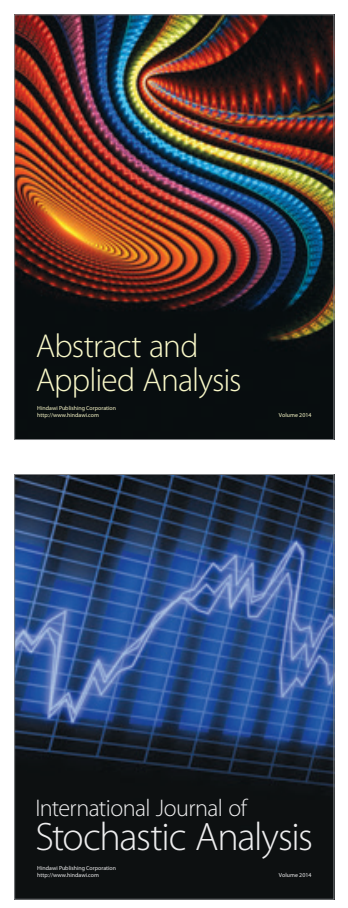

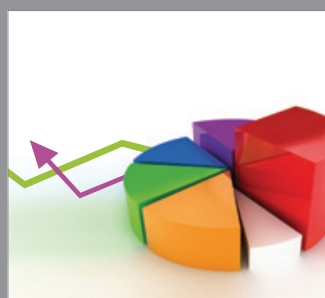

ournal of

Probability and Statistics

Promensencen
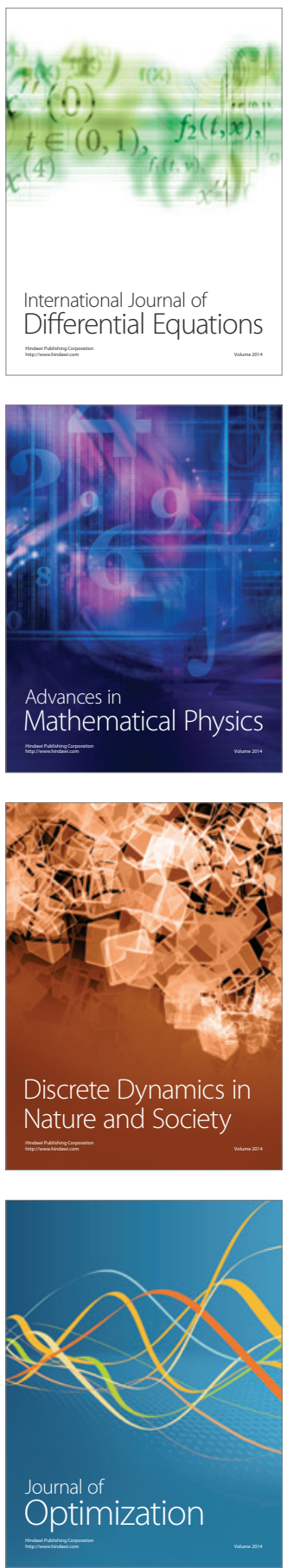